\title{
Research on Application of ETAP in Ship electric propulsion system
}

\author{
Kai Liu ${ }^{1 *}$, Jing Chen ${ }^{1}$ \\ ${ }^{1}$ Wuhan Institute of Marine Electric Propulsion, Wuhan, China.
}

\begin{abstract}
This article takes a ship electric propulsion system as a specific example, using ETAP to establish a simulation model and short-circuit current calculation. Through the calculation results, the coordination setting of the circuit breaker and the comparison with the COMPASS calculation results are carried out, and finally the selective protection and coordination analysis of the power system are completed.
\end{abstract}

\section{Introduction}

In the ship power system, short circuit is the most common and serious fault, which refers to the direct metallic connection between phase and phase or between phase and ground. There are four main types: three-phase, twophase, single-phase grounding and two-phase grounding short-circuit, in which only three-phase short circuit is a symmetrical short circuit.

In order to ensure that short-circuit fault can be quickly and effectively removed, it is necessary to accurately estimate the current value of each short-circuit point, so as to reasonably select the protection method and protection device. Based on an engineering project example, this paper uses ETAP software to model and simulate the ship electric propulsion system and realizes the calculation and analysis of short-circuit current and selective protection coordination.

\section{Software introduction}

ETAP is a powerful power analysis software, launched by the American OTI company in 1996. It is widely used in the electric power industry, with functions such as power flow analysis, short circuit calculation, protection setting, harmonic analysis, etc., providing convenience for electrical system calculations. It has the following characteristics: (1) Provide an easy-to-operate interface that can quickly model the power system;(2) With virtual operation, the results are displayed in the form of graphics and reports;(3) Powerful design and calculation and analysis functions;(4) Contains various short-circuit standards such as ANSI and IEC for selection.

The technicians carry out the preliminary design according to the technical requirements, and use ETAP to check the rationality of the equipment model, power distribution, and relay protection, etc., which can effectively reduce the project cost and improve work efficiency.

\section{Simulation model}

ETAP software is very convenient to establish a single-line diagram of a power system according to the actual wiring diagram. What need to do is dragging and dropping each component to the interface according to the wiring diagram and the steps are as follows:

(1) According to the actual operating conditions, determine the maximum and the minimum short-circuit current calculation model, and sometimes the emergency short-circuit current and the short-time parallel connection calculation model are needed;

(2) Determine the short-circuit current value at a specific point in the network, generally including: generator outlet near the main switchboard, main busbar, emergency switchboard, and district switchboard, the secondary of the power and lighting transformer, the distribution board bus of the power, and the outlet end of the large motor near the busbar;

(3) The short-circuit current under the maximum working condition is used to check the segmentation capacity, making capacity of the circuit breaker, and the electrodynamic stability and thermal stability of the busbar;

(4) Perform relay protection setting coordination and draw out the setting coordination time-current curve of each protection and adjust the protection setting value online in combination with the short-circuit current.

When drawing the one-line diagram model according to the actual wiring diagram, it is necessary to pay attention to the influence of the variable speed drive (VSD) on the short circuit fault. Since the pulse width modulation (PWM) converter driver will be disconnected from the fault within a few seconds and will not provide any current for the fault, the impact of the branch on the short-circuit current is usually omitted.

\footnotetext{
*Corresponding author's e-mail: lu_yf.sy@gx.csg.cn
} 


\section{IEC60909 short-circuit calculation}

The IEC 60909 standard specifies four types of shortcircuit faults. Use the results of software calculations to complete electrical equipment short-circuit, thermal stability verification, relay protection settings and checks the rated operation and interruption capacity of the protection equipment under fault current conditions.

For the marine electrical system, the main focus is on the check of the circuit breaker. Therefore, this article takes a three-phase short circuit as an example for the calculation.

After the circuit breaker is selected, the short-circuit making capacity and breaking capacity of the selected protection electrical are checked. From the calculation results, it can be seen whether the selected circuit breaker meets the requirements, if not, the parameters can be adjusted to ensure the safety and reliability of the system.

\section{IEC61363 short circuit calculation}

The IEC61363 transient short-circuit current calculation expresses the waveform of the fault current in the form of a time function, which takes into account the factors including synchronous motor sub-transient impedance, transient impedance, transient time constant, sub-transient time constant, etc.

This detailed calculation model provides accurate short-circuit current estimation for the determination of the capacity of protective equipment and the coordination of relay protection for ships. However, it should be noted that IEC61363 can only calculate three-phase (balanced) short-circuit current, and for simplicity, usually the peak current is the current at $\mathrm{t}=\mathrm{T} / 2=0.01 \mathrm{~s}(\mathrm{f}=50 \mathrm{~Hz})$.

In the summary report, we can see the sub-transient, transient and steady-state currents of each short-circuit point; Figure 1 show the changes in the fault current at each short-circuit point with data and graphs. Therefore, the selected protection electrical short-circuit making capacity and breaking capacity can be further checked and verified according the requirements of CCS.

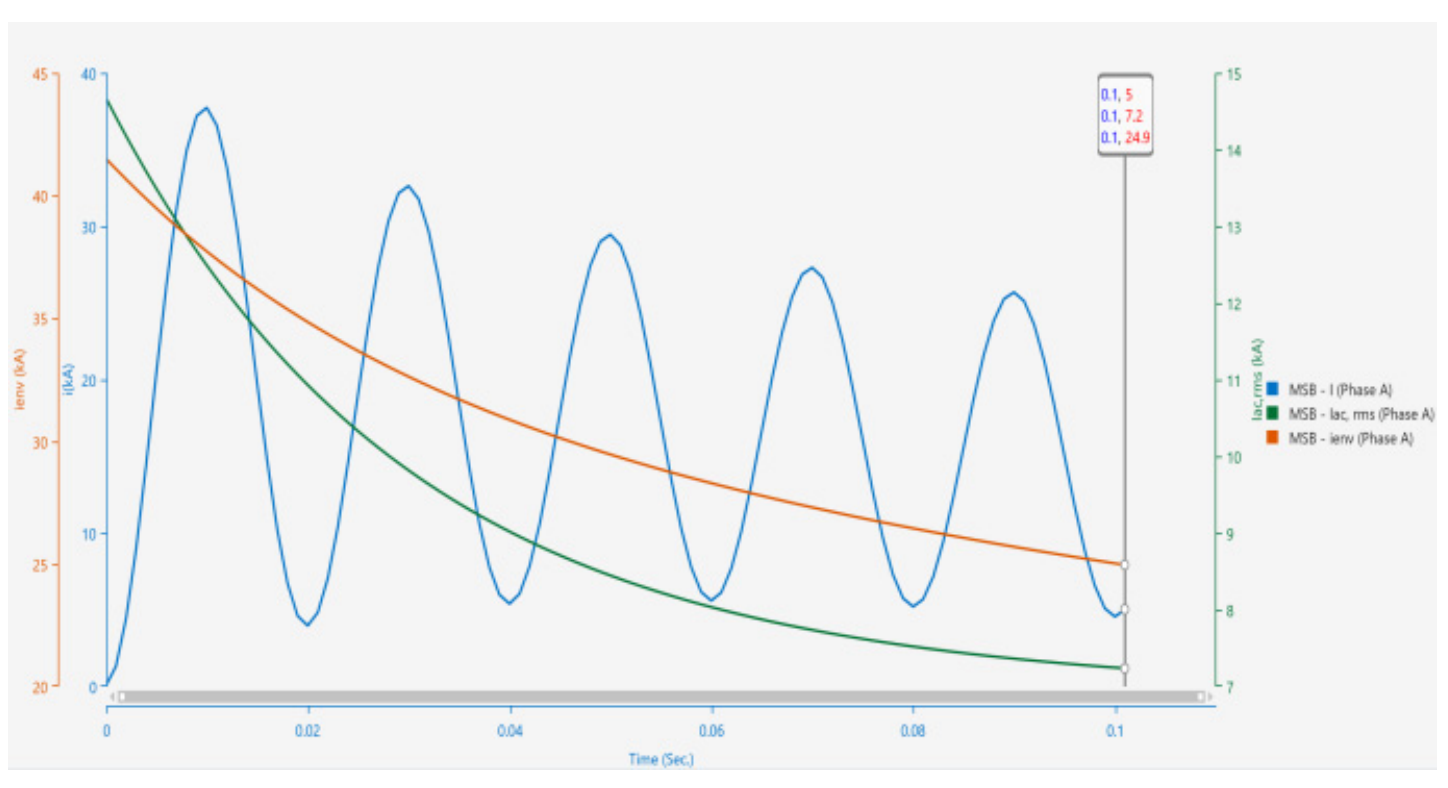

Figure 1. IEC61363 MSB current waveform

\section{Compare with COMPASS results}

Table 1. Comparison of IEC61363 and COMPASS results

\begin{tabular}{|c|c|c|c|c|}
\hline \multirow{2}{*}{} & \multicolumn{2}{|c|}{ IEC63136 } & \multicolumn{2}{c|}{ COMPASS } \\
\cline { 2 - 5 } & ip & Iac & ip & 27.4807 \\
\hline ESB1 & 47.229 & 27.744 & 45.2961 & 57.4807 \\
\hline ESB1Transf1 & 29.479 & 19.735 & 45.2961 & 5.5722 \\
\hline ESB2 & 8.4125 & 5.5404 & 10.2731 & 12.7218 \\
\hline MSB1 & 35.265 & 10.758 & 34.4297 & \\
\hline
\end{tabular}




\begin{tabular}{|c|c|c|c|c|}
\hline MSB2 & 78.5 & 34.998 & 75.4233 & 35.6431 \\
\hline MSB3 & 61.713 & 31.691 & 59.1933 & 31.8567 \\
\hline
\end{tabular}

\section{Star view for relay protection setting}

Star view is a fully integrated system protection device coordination module. By inputting the setting value, the setting coordination curve of each protection can be drawn and combined with the short-circuit current to adjust the protection setting value online. Utilizing intelligent oneline diagrams, comprehensive protection device library and integrated three-dimensional database, star view enables electrical engineers to implement protection device coordination analysis conveniently and efficiently. As for fault simulation verification can help power system engineers and planners quickly realize possible design solutions and make a wise decision to improve the reliability of the system, increase the stability of the system, and save costs.

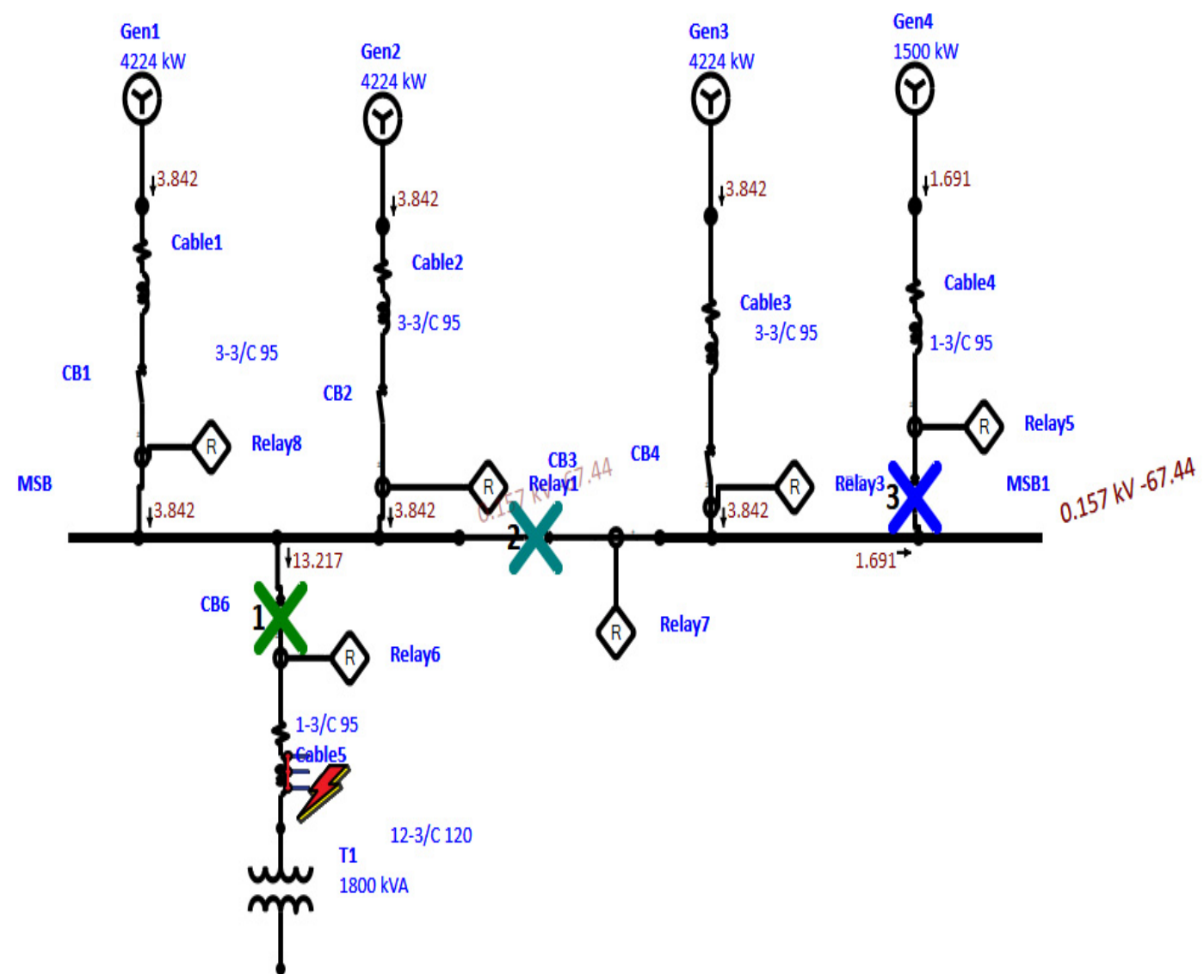

Figure 2.Fault simulation verification

Figure 2 shows the relay action sequence of simulation during short circuit. Therefore, by a shortcircuit fault simulation of ETAP, selecting the shortcircuit point and the fault type, it can visually observe the action time and sequence of each circuit breaker after a short-circuit fault, thereby verifying whether the selective protection of the circuit breaker is coordinated.

\section{Summary}

Combined with an example, this article introduces the short-circuit current of the ship electric propulsion system using ETAP software, which can complete short-circuit calculation, protection setting coordination, and form a unified calculation analysis report, verify the design of the electric propulsion system, and greatly reduce the workload of calculation and analysis. .It can visually observe the action time and sequence of each circuit breaker after the occurrence of a short-circuit fault, thereby verifying whether the selective protection of the circuit breaker is coordinated.

\section{References}

1. Pan Yao. Application of ETAP power software in relay protection setting and protection coordination of medium voltage power supply and distribution system in sintering plant[J]. Engineering 
Construction, 2018, 50(05): 46-51.

2. Ke Changguo, Wu Chunji, Li Lihong. Simulation analysis of electric propulsion system based on ETAP[J]. Ship Electric Technology, 2016, 36(02): 71$74+77$.

3. Liu Sen, Wang Yang, Zhou Yingying. Application of ETAP software in short-circuit calculation and relay protection setting of cement plants[J]. China Cement, 2012(06): 70-72.

4. Zheng Xiaowei. Short-circuit current calculation of low-voltage power grid based on ETAP software[J]. Science Technology and Innovation, 2019(07): 52$53+55$. 